\title{
BMJ Open Health and social interventions to restore physical function of older adults post-hip fracture: a scoping review
}

\author{
Abeer Omar (iD , ${ }^{1}$ Alexia Cumal, ${ }^{2,3}$ Shirin Vellani (D) , ${ }^{2,4}$ Alexandra Krassikova (D) ,2,5 \\ Julie Lapenskie, ${ }^{6,7}$ Melanie Bayly, ${ }^{8}$ Vivian A Welch (D) , ${ }^{6,9}$ Elizabeth Ghogomu, ${ }^{6}$ \\ Andrea laboni, ${ }^{2,10}$ Katherine S McGilton ${ }^{2,3}$
}

To cite: Omar A, Cumal A, Vellani S, et al. Health and social interventions to restore physical function of older adults post-hip fracture: a scoping review. BMJ Open 2021;11:e053992. doi:10.1136/ bmjopen-2021-053992

- Prepublication history and additional supplemental material for this paper are available online. To view these files, please visit the journal online (http://dx.doi.org/10.1136/ bmjopen-2021-053992).

Received 08 June 2021 Accepted 05 October 2021

Check for updates

(c) Author(s) (or their employer(s)) 2021. Re-use permitted under CC BY-NC. No commercial re-use. See rights and permissions. Published by BMJ.

For numbered affiliations see end of article.

Correspondence to

Dr Abeer Omar;

abeeromar@trentu.ca

\section{ABSTRACT}

Objectives Despite inpatient rehabilitation, many older adults post-hip fracture have difficulty returning to their prefracture levels of function and activity. This scoping review aims to identify interventions for communitydwelling older adults discharged from inpatient rehabilitation and examine the function and activity outcomes associated with these interventions.

Design This scoping review followed Arksey and 0'Malley's five-stage framework.

Data sources We searched MEDLINE, CINAHL, PsycINFO, EMBASE and Ageline electronic databases for Englishlanguage articles published between January 1946 and January 2020.

Eligibility criteria We included studies with health and social interventions involving community-dwelling older adults and their caregivers after hip fracture and inpatient rehabilitation. The interventions were selected if initiated within 60 days post-hip fracture surgery.

Data extraction and synthesis Two independent reviewers screened abstracts and full texts and extracted the data based on the inclusion criteria. A third reviewer adjudicated any disagreement and collated the extracted data.

Results Of the 24 studies included in the review, most studies $(79 \%)$ used exercise-based interventions, over half $(63 \%)$ included activities of daily living training and/ or home assessment and environment modification as intervention components, and three studies used social intervention components. Over half of the interventions $(58 \%)$ were initiated in the inpatient setting and physiotherapists provided $83 \%$ of the interventions. Only seven studies (29\%) involved tailored interventions based on the older adults' unique needs and progress in exercise training. Six studies (25\%) enrolled patients with cognitive impairment, and only one study examined caregiverrelated outcomes. Exercise-based interventions led to improved function and activity outcomes. 29 different outcome measures were reported.

Conclusion While exercise-based multicomponent interventions have evidence for improving outcomes in this population, there is a paucity of studies, including social interventions. Further, studies with standardised outcome measures are needed, particularly focusing on supporting caregivers and the recovery of older adults with cognitive impairment.
Strengths and limitations of this study

- This scoping review used a systematic approach by application of a five-stage methodological framework.

- Our review included the majority of randomised controlled trials published between 1945 and 2020.

- This review included intervention studies published in English only, and other studies published in different languages may include other health and social interventions for older adults post-hip fracture.

- We searched only five databases and excluded the grey literature.

\section{INTRODUCTION}

Older adults who sustain a hip fracture are often medically complex, often have pre-existing functional dependence and frequently present with dementia. ${ }^{1}$ Only about $40 \%-60 \%$ of patients with hip fracture recover their prefracture level of function and mobility ${ }^{2}$ with most recovery occurring in the first 6 months. ${ }^{3}$ Evidence suggests that rehabilitation of older adults post-hip fracture surgery may lead to improvement of outcomes in this population. ${ }^{14}$ However, there is an unmet need of rehabilitation services globally, despite it being recognised as essential in the continuum of care. ${ }^{5}$

An examination of care pathways in Ontario, Canada for patients with hip fracture discharged from acute care revealed that the majority of patients are discharged either to inpatient rehabilitation, which offers shortterm intensive rehabilitation, or directly to the community, followed by communitybased rehabilitation. ${ }^{6}$ Although older adults who received additional home health services are less likely to be hospitalised or institutionalised than those who received only inpatient rehabilitation, ${ }^{7}$ the services and supports provided in the community may not be sufficient to optimise health and prevent 
functional decline over time. McGilton et al completed a 6-month unpublished project in 2015 entitled 'An in-patient rehabilitation model of care targeting cognitive impairment for patients post-hip fracture surgery: A demonstration project' and found that $26 \%$ and $21 \%$ experienced a decline in physical functioning 3 and 6 months after discharge, respectively. Furthermore, an examination of home care services provided to persons with hip fracture revealed that only half of this population receive visits from physiotherapists and even less from occupational therapists. ${ }^{8}$

In the current review, a conceptual framework of geriatric rehabilitation outcomes proposed by Demers et al is used to define activity as mobility, basic and instrumental activities of daily living (IADL) and leisure activities; whereas functioning can be described as physical, psychological, social functioning and caregiver status. ${ }^{9} \mathrm{~A}$ meta-analysis of home-based rehabilitation exercise interventions for older adults with hip fractures showed that home-based rehabilitation improved mobility and ability to carry out ADL. ${ }^{10}$ A separate meta-analysis of rehabilitation interventions demonstrated that offering extended exercise at home or in the community can improve the functional abilities of older adults with hip fracture. ${ }^{11}$ A systematic review of community-based rehabilitation interventions for persons with cognitive impairment who sustain a hip fracture suggests that community-based interventions may improve mobility and physical function, and ADL performance in older adults with dementia. ${ }^{12}$ Moreover, the American Physical Therapy Association recommends that older adults with hip fracture receive strength, balance, functional and gait training as part of home care or community-based intervention. ${ }^{13}$ Still, solely exercisebased interventions may not be sufficient.

Multiple pre-existing factors, such as multimorbidity, cognitive impairment, depression, poor nutritional status and lack of social support, can impact functional recovery, necessitating the implementation of multicomponent interventions that facilitate recovery in older adults postdischarge from inpatient rehabilitation after hip fracture surgery. ${ }^{14}$ There is a need to determine the various components of health and social interventions (henceforth referred to as interventions) that can reduce the risk of further decline in activity and function once older adults are discharged home from inpatient rehabilitation post-hip fracture surgery. Therefore, this scoping review aims to identify the health and social interventions associated with function and activity outcomes once older adults are discharged home from inpatient rehabilitation post-hip fracture surgery.

\section{METHODS}

A scoping review methodology was selected to map different types of interventions designed to restore older adults' function and activity post-hip fracture, in addition to the range of measured outcomes. This review followed the framework stages outlined by Arksey and O'Malley ${ }^{15}$ and advanced by Levac et al. ${ }^{16}$ This review also followed the Preferred Reporting Items for Systematic Reviews and Meta-Analyses (PRISMA) extension for Scoping Reviews statement. ${ }^{17}$

This scoping review examines health and social interventions aiming to optimise physical functioning and activity in older adults postdischarge from inpatient rehabilitation settings. Health needs are defined as those related to prevention, management, treatment or control of illness or disability provided by health professionals. ${ }^{18}$ In comparison, social needs are unmet because of physical or mental impairments. For example, lack of social support, access to community services, nutrition and home safety can significantly impact the individuals' health. According to the WHO International Classification of Functioning, Disability and Health (ICF) framework, ${ }^{19}$ mental and psychological factors can interact with health conditions to impact on a person's functioning and recovery. Health interventions are defined by the authors and may include exercise, nutrition, assistance with basic ADLs and promoting physical activity in outpatient clinics or community home visits. On the other hand, social interventions emphasise increasing access to emotional, instrumental and informational support for older adults and their caregivers. It may involve access to assistance with IADLs (eg, home cleaning, transportation, meal preparation, shopping, and medication management), social work and caregiver support services. Health and social interventions are not mutually exclusive and often overlap as both provide support to help with the rehabilitation of older adults ${ }^{18}$; both will henceforth be referred to as interventions.

\section{Framework stage 1: identifying the research questions}

This scoping review examines the peer-reviewed research on interventions provided to community-dwelling older adults and caregivers following discharge from inpatient rehabilitation post-hip fracture surgery. This scoping review asks the following questions: (1) What health and social interventions are available for older adults post-hip fracture? and (2) What functional and activity outcomes are associated with health and social interventions for older adults and their caregivers after discharge from inpatient rehabilitation post-hip fracture?

\section{Framework stage 2: identifying relevant studies}

An exhaustive search in MEDLINE, CINAHL, PsycINFO, EMBASE and Ageline databases was conducted that covered the period between January 1946 and 23 January 2020. The year 1946 was chosen to have an exhaustive search strategy. Based on the aims of the review, three keywords were used: 'older adults,' 'hip fracture' and 'health and social services interventions' in preparing the search strategy, in consultation with an information specialist. The search strategy was translated according to each database platform's command language, controlled vocabulary and appropriate search fields. The search strategies for all databases are available for reference in 
online supplemental appendix A, and MEDLINE strategies are used as an example. The search strategy was limited to peer-reviewed publications in English.

\section{Framework stage 3: study selection}

The current scoping review included randomised controlled trials (RCTs), as well as before and after control, retrospective, prospective and longitudinal studies if they met the following criteria: (1) written in English; (2) involved community-dwelling older adults aged 65 years or older following discharge from inpatient rehabilitation post-hip fracture surgery and/or their caregivers; (3) reported outcome data on function and mobility and (4) included at least one health and/ or social intervention that began within 60 days of the hip fracture surgery. Sixty days limitation was selected because this period captures 10-20 days of inpatient rehabilitation (the standard length of inpatient rehabilitation in Canada), plus the recommended standard treatment for outpatient rehabilitation that is usually provided within the 4-6weeks period following discharge from rehabilitation. ${ }^{20}$ We excluded articles that involved patients having elective hip surgeries. The RCTs, before and after control, retrospective and longitudinal studies were included as they reported on the influence of interventions on outcomes, which is one of this scoping review's goals. English-language studies were chosen as our authors are English-speaking.

EndNote V.X8 reference manager was used to collate retrieved records. After systematic deduplication, the final unique set of records was imported into Covidence. This web-based software program streamlines the screening, study selection and data extraction for reviews. ${ }^{21}$ Titles and abstracts were screened by two reviewers (AO, SV, $\mathrm{KSM}$, JL, MB and $\mathrm{HC}$ ) independently based on the inclusion criteria. Then, two reviewers assessed each full-text article (SV, AO, MB, AI, JL, KSM and $\mathrm{HC}$ ). The reference lists of all included articles and relevant reviews were also screened to identify additional related work. Any cases of disagreement between reviewers were adjudicated by the senior lead investigator (KSM).

\section{Framework stage 4: data extraction}

Microsoft Excel was used to extract the study data. A prepiloted Excel form included (1) details of the study (country, sample size, study design, first author, publication year); (2) patient characteristics (sex, age, marital status, race, income, comorbid conditions, prefracture functional status); (3) caregiver characteristics (sex, age, relationship to the patient); (4) intervention characteristics (dose, duration, frequency, steps and components of interventions); (5) outcome characteristics (older adult and caregiver outcome types, instruments used, frequency of measurements) and (6) results related to the primary outcome. Two reviewers extracted each selected study (KSM, AO, SV, MB, AI, JL, HC and AC). After the extraction of all included studies, a third reviewer (AO)
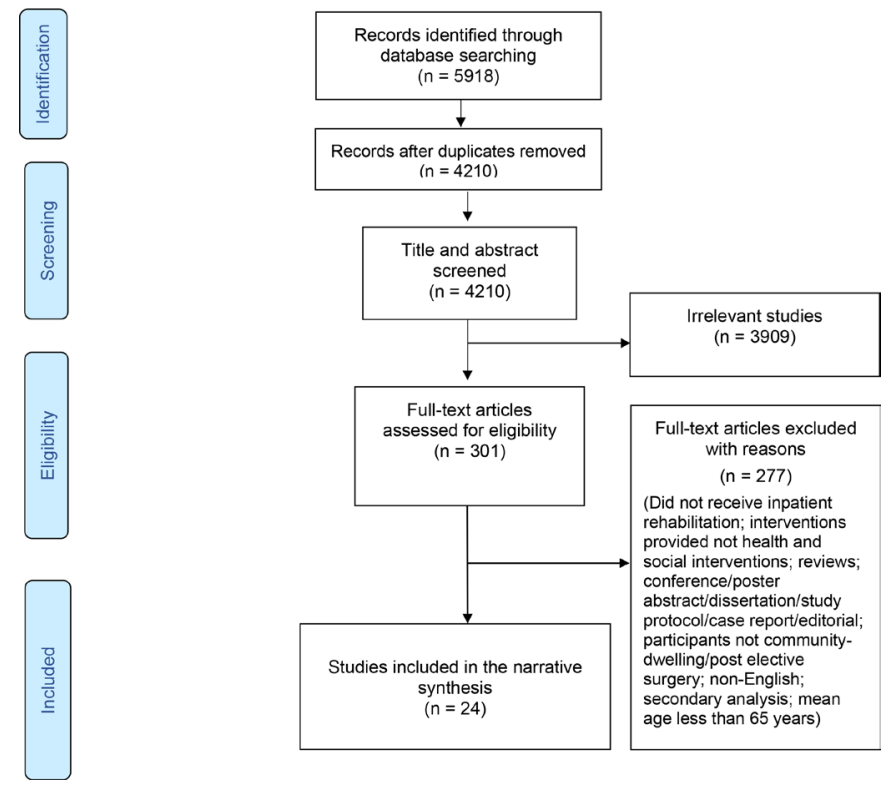

Figure 1 PRISMA flow diagram. PRISMA, Preferred Reporting Items for Systematic Reviews and Meta-Analyses.

collated the data into one Excel file and compared data extraction accuracy for each study.

\section{Framework stage 5: collating, summarising and reporting the results}

Extracted data were critically analysed, and outcomes reported in studies were categorised according to Demers et als conceptual framework of geriatric rehabilitation outcomes. ${ }^{9}$ Demers et al categorised function and activity outcomes into activity domain and functioning domain. ${ }^{9}$ The activity domain includes mobility activities, basic ADLs, independent activities and leisure activities. ${ }^{9}$ The functioning domain includes physical, psychological and social functioning, and caregiver status and available resources. We summarised the current state of knowledge, described intervention components and measured outcomes in a narrative synthesis.

\section{Patient and public involvement}

There was no patient involved.

\section{RESULTS}

The databases search identified 5918 records. Following deduplication, a final set of 4210 titles and abstracts were screened, of which 301 abstracts were eligible for full-text review. Based on the a priori inclusion criteria, 24 studies were included in the final synthesis (see PRISMA diagram, figure 1). Cohen's Kappa for inter-rater reliability (IRR) between the reviewers screening for abstracts and the full text is retrieved from Covidence web-based software. The average IRR between reviewers screening full-text articles is 0.42 , which is considered moderate. ${ }^{22}$ The average IRR of 0.29 for the title and abstract screening reflects fair reliability. 


\section{Characteristics of included studies}

The current review included a final number of 24 studies. The majority $(71 \%)$ of those studies were conducted in Europe and North America, ${ }^{23-38} 16 \%$ in Asia ${ }^{39-41}$ and $13 \%$ in Australia. ${ }^{42-44}$ The study designs consisted of RCTs $(n=16),{ }^{23-27} 2930323536 \quad 3841 \quad 43-46$ quasi-RCTs $(\mathrm{n}=2),{ }^{28} 42$ feasibility RCTs $(\mathrm{n}=2),{ }^{37}{ }^{39}$ prospective cohort studies $(n=3)^{33} 3440$ and observational pre-post $(n=1) .^{31}$ The sample sizes ranged from $26^{26}$ to $443^{34}$ older adults discharged from inpatient rehabilitation programmes post-hip fracture surgery. Only one study included caregiver participants. ${ }^{37}$ Table 1 displays the detailed study characteristics.

\section{Characteristics of participants}

The mean age of patient participants ranged from $73^{41}$ to 84.3 years. ${ }^{25}$ The percentage of females in the studies ranged from $45 \%^{23}$ to $100 \%,{ }^{27} 283542$ with four studies including only female participants. ${ }^{27} 283542$ Six $(25 \%)$ studies enrolled patients with cognitive impairment. 303334364042 The average number of comorbidities ranged from 2.7 to $3.4 .^{27} 44$

\section{Health and social interventions offered to older adults post- hip fracture}

Over half of the interventions $(58 \%)$ were initiated in the inpatient setting and continued at

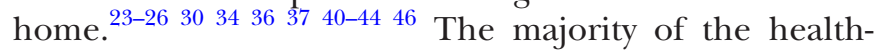
care providers who conducted the intervention were physiotherapists $(83 \%),{ }^{23-27} \quad 29 \quad 30 \quad 33 \quad 34 \quad 36-41 \quad 43-47$ either alone, ${ }^{242933394143-4547}$ with physiotherapy assistants or technical instructors, ${ }^{26} 37$ or combined with other providers such as nurses, medical doctors; geriatricians, psychiatrists and/or occupational therapists. ${ }^{232527303436384046}$ The intervention duration ranged from $1^{42}$ to 12 months. ${ }^{25} 293544-46$ We identified interventions with 1 to 12 different components, and most of the studies $(n=17)$ used a combination of intervention components (table 2). ${ }^{23-30323638404143-46}$ The most frequently used components were exercise $(\mathrm{n}=19),{ }^{23-25293032-4143-46}$ ADL training $(\mathrm{n}=9)^{2728303840414344}$; geriatric assessment $(n=7)^{27283036384446}$ and home assessment and fall prevention $(n=7) .{ }^{26-29} 384446$ No intervention components were explicitly aimed at improving the experience or outcomes of caregivers.

The interventions in this review were classified into two major distinguishing categories: (1) exercise-based interventions and (2) non-exercise-based interventions. If one component of the intervention included physical exercise, it was categorised as an exercise-based intervention. A brief overview of the interventions is presented below. See online supplemental appendices B and C for additional details of the interventions' characteristics such as mode of delivery, provider, duration, adherence and control groups for each study.

\section{Exercise-based interventions}

Nineteen studies of exercise-based interventions consisted of different activities such as muscle strength training, balance training and weight-bearing exercises (online supplemental appendix B). Out of those 19 studies, over one-third of studies' interventions $(37 \%$, $\mathrm{n}=7$ ) were described by the study investigators as tailored to the individual's unique needs and progress in exercise training. 24293037384145

Five studies had exercise training as the sole intervention. ${ }^{33} 35373943$ Of those five, four studies had the physiotherapist (PT) as the facilitator, ${ }^{33} 373943$ and in one study, the exercise was delivered by an exercise trainer. ${ }^{35}$ The majority of interventions were initiated on discharge either from acute care or rehabilitation facility. ${ }^{33} 3739$ Intervention length varied from 6 weeks ${ }^{33}$ to 12 months. ${ }^{35}$ Two separate interventions that were delivered by a PT twice a week for 6 weeks showed significant improvements in balance. ${ }^{33}{ }^{39}$ Statistically significant changes in exercise behaviour were reported in one study where the exercise was delivered by an exercise trainer for 12 months. ${ }^{35}$ One study examined the association between progressive lower limb strength training delivered by a PT and gait speed, mobility score, walking ability, muscle strength and endurance, all of which showed statistically significant improvements. ${ }^{33}$ Older adults with hip fracture that had exercise delivered by a PT and OT immediately since surgery showed statistically significant improvements in walking ability and mobility score. ${ }^{34}$ One intervention that involved six therapy sessions delivered by a PT over the course of 3 months showed no significant improvements in ADLs. ${ }^{37}$

Nineteen studies included exercise-based interventions, ${ }^{23-25} 293032-3436-46$ with 14 of them being multicomponent. ${ }^{23-25} 2930323638404143-46$ Of these, seven were tailored to the unique characteristics of the patients. ${ }^{23} 293037384145$ The most common component with exercise was ADL training $(\mathrm{n}=7) .^{29} 303840414344$ Table 2 illustrates other intervention components combined with exercise such as osteoporosis treatment $(n=6)$, nutrition $(n=5)$, home assessment and fall prevention $(n=4)$, social support $(n=3)$, depression management $(n=2)$, medication review $(n=2)$ and vision care $(n=1)$. Only 4 of 19 studies involved multidisciplinary team including PT, OT, nurses and physicians, to deliver the interventions. ${ }^{23} 303646$ Length of exercise component ranged from 3 weeks postrehabilitation discharge ${ }^{38}$ to 12 months $^{25} 29404546$; and were generally managed by physiotherapists. Several studies involved in-person visits at patients' home by team members to provide one on one treatment and optimise adherence. $^{34363840}$ There was a wide range of outcomes measured using a variety of tools. See table 3 for the outcome measures, tools used and if they were statistically significant.

\section{Non-exercise-based interventions}

Five studies had non-exercise-based intervention components. ${ }^{26-283142}$ Two studies combined ADL training, osteoporosis management, home assessment and environment modification and geriatric assessment in addition to the usual multidisciplinary rehabilitation that included 


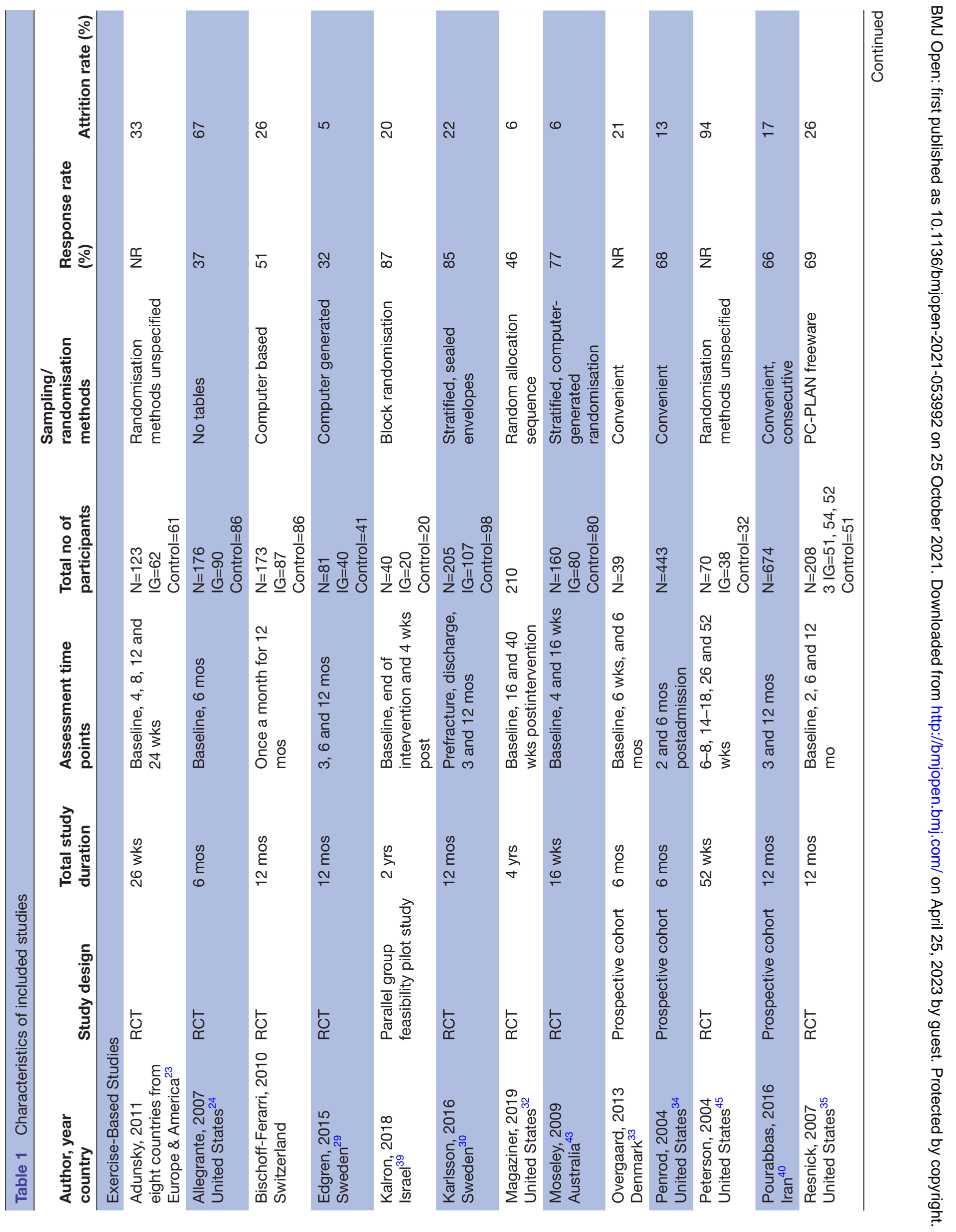




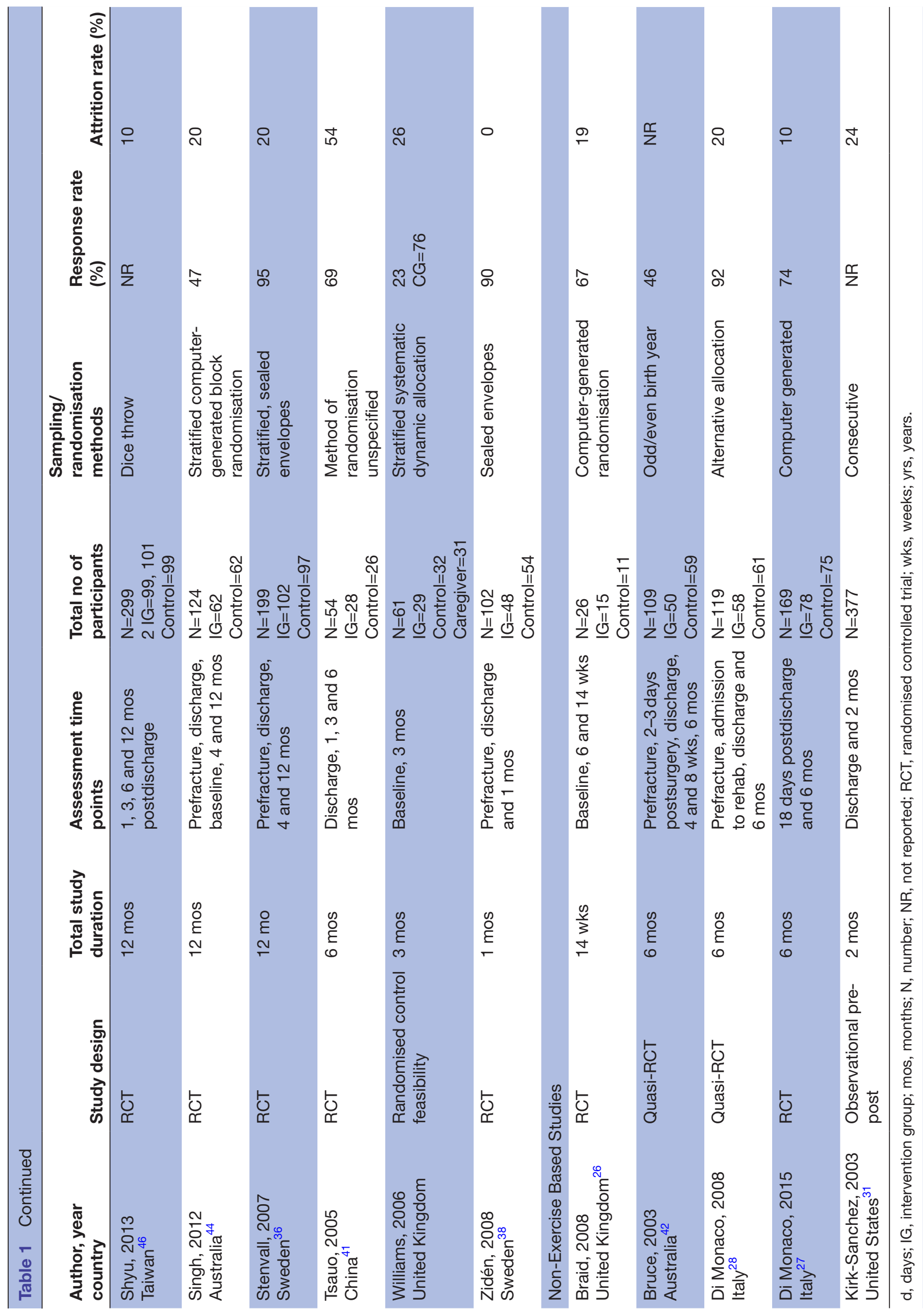

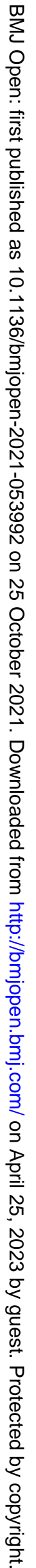




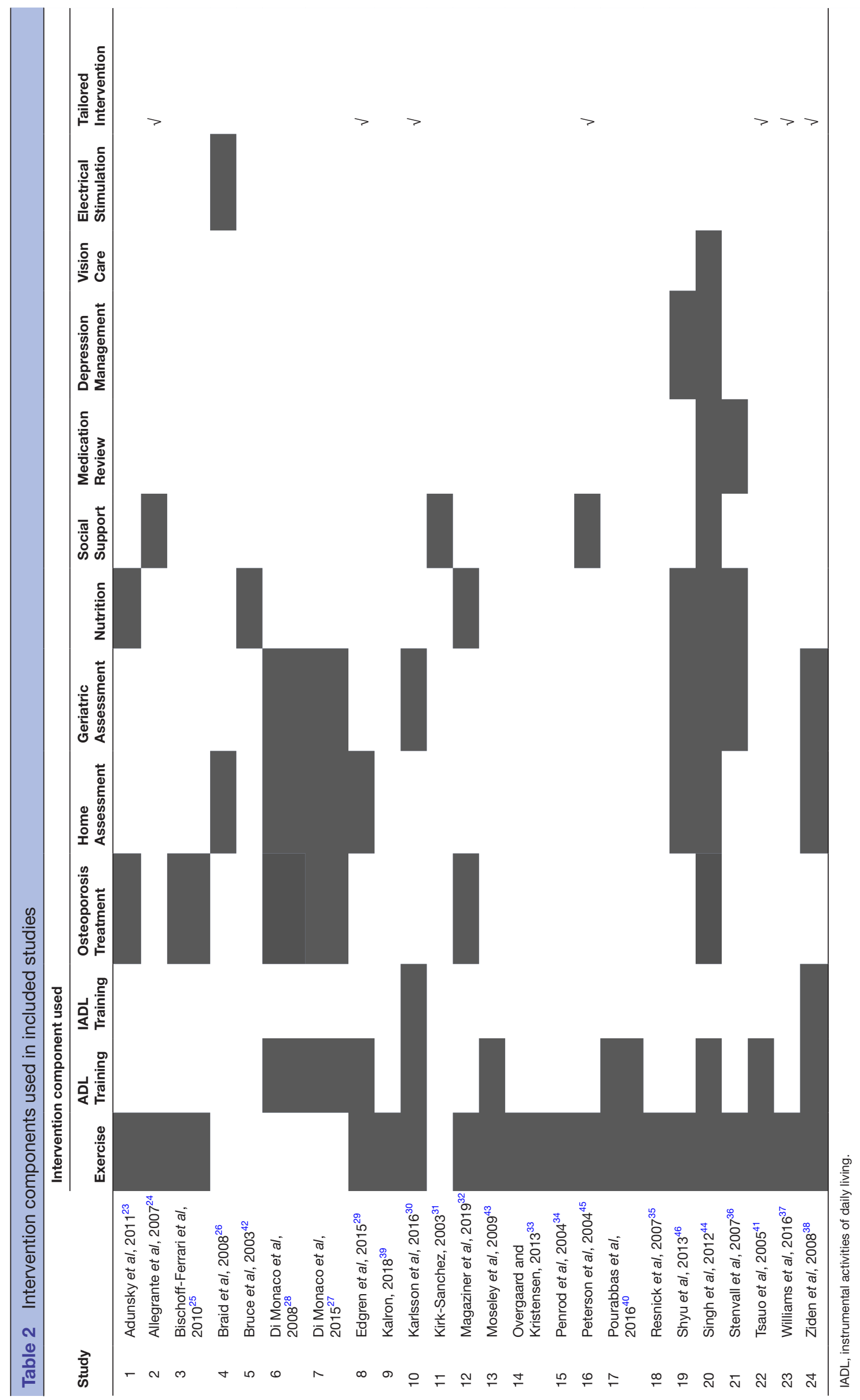

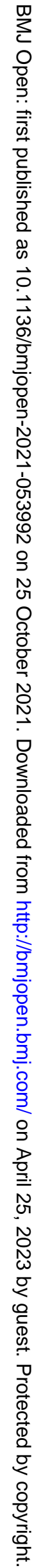


Table 3 Outcome measures in included studies

\begin{tabular}{|c|c|c|}
\hline Outcome/construct & Measurement tool & Studies statistical significance \\
\hline \multicolumn{3}{|l|}{ Activity domain } \\
\hline Mobility & $\begin{array}{l}\text { 1. Timed-Up-and-Go Test } \\
\text { 2. Gait Speed Test } \\
\text { 3. New Mobility Score } \\
\text { 4. Locomotion Subscale of Functional Independence } \\
\text { Measure } \\
\text { 5. } 6 \text { min Walk Test/50-foot walk/10 min walk } \\
\text { 6. Self-reported use of walking device indoors and } \\
\text { outdoors } \\
\text { 7. Sit-to-Stand Test } \\
\text { 8. SPPB } \\
\text { 9. Elderly Mobility Scale } \\
\text { 10. AM-PAC } \\
\text { 11. S-COVS } \\
\text { 12. Distance walked in } 6 \text { min } \\
\text { 13. mPPT }\end{array}$ & $\begin{array}{l}\text { 1. }(26)^{\star}(34)^{\star}(46)^{\star} \\
\text { 2. }(24)^{\star}(27)(31)(33) \dagger(34)^{\star}(40)^{\star}(42)(44) \\
(46)^{\star} \\
\text { 3. }(31)(33)(34)^{\star}(35)^{\star}(37)(46) \\
\text { 4. }(35)^{\star}(41)^{\star} \\
\text { 5. }(34)^{\star}(38)(46)^{\star} /(33) /(40)^{\star} \\
\text { 6. }(31)(34)^{\star}(37)(41)^{\star}(45)^{\star} \\
\text { 7. }(38)(40)^{\star}(44)^{\star} \\
\text { 8. }(24)^{\star}(33) \dagger \\
\text { 9. }(27) \\
\text { 10. }(24) \\
\text { 11. }(37)^{\star} \\
\text { 12. }(33) \dagger \\
\text { 13. }(33) \dagger\end{array}$ \\
\hline IADLs & $\begin{array}{l}\text { 1. Assessment of Living Skills and Resources } \\
\text { 2. Instrumental Activity Measure } \\
\text { 3. Staircase of ADL, that measured ADLs using the } \\
\text { Katz Index as well as IADLs } \\
\text { 4. Physical Disability questionnaire on perceived } \\
\text { difficulties in performing } 6 \text { ADLs and } 8 \text { IADLs }\end{array}$ & $\begin{array}{l}\text { 1. }(45) \\
\text { 2. }(30)(37)^{\star}(39)^{\star}(45)(47)^{\star} \\
\text { 3. }(43) \\
\text { 4. (30) }\end{array}$ \\
\hline
\end{tabular}

Functioning domain

1.Physical functioning

\begin{tabular}{|c|c|c|}
\hline Balance & $\begin{array}{l}\text { 1. Rate of falls } \\
\text { 2. Tandem test } \\
\text { 3. Falls efficacy scale } \\
\text { 4. SPPB } \\
\text { 5. Functional Reach Test } \\
\text { 6. Timed-Up-and-Go Test } \\
\text { 7. National Health and Ageing Trends Study }\end{array}$ & $\begin{array}{l}\text { 1. }(24)(26)^{\star}(28)(29)^{\star}(30) \\
\text { 2. }(34)^{\star} \\
\text { 3. }(38)(39) \\
\text { 4. }(24)^{\star}(33) \dagger \\
\text { 5. }(46) \\
\text { 6. }(26)^{\star}(34)^{\star}(46)^{\star} \\
\text { 7. }(33)\end{array}$ \\
\hline Endurance & $\begin{array}{l}\text { 1. SF-36 Physical Health } \\
\text { 2. Outcome Expectations for Self-efficacy for Walking/ } \\
\text { Exercise Scale } \\
\text { 3. Muscle strength } \\
\text { 4. Range of motion } \\
\text { 5. Nottingham Power Rig }\end{array}$ & $\begin{array}{l}\text { 1. }(25)(34)^{\star} \\
\text { 2. }(36)^{\star} \\
\text { 3. }(24)(26)^{\star}(27)(33)^{\star}(34)^{\star}(38)(39)^{\star}(42) \\
(44)(46)^{\star} \\
\text { 4. }(42) \\
\text { 5. }(27)\end{array}$ \\
\hline Comfort & 1. Health-related quality of life & 1. $(42)^{\star}$ \\
\hline 2.Caregiver status & 1. Caregiver Strain Index & 1. (38) \\
\hline
\end{tabular}


*Statistically significant.

†Improvement seen in intervention and control groups.

ADLs, activities of daily living; AM-PAC, Activity Measure for Post-Acute Care; IADLs, instrumental ADLs; ; IGF-1, insulin like growth factor; mPPT, modified physical performance test; NHANES, National Health and Nutrition Examination Survey; OP, osteoporosis; S-COVS, Swedish Physiotherapy Clinical Outcome Scale; SF, Short Form; SPPB, Short Physical Performance Battery.

exercise and fall prevention measures. ${ }^{27}{ }^{28}$ The above multicomponent intervention was delivered via single home visit by an OT, ${ }^{28}$ or a single phone call. ${ }^{27}$ One study involved a nutritional supplement ${ }^{42}$; one assessed social support ${ }^{31}$ and one used electrical stimulation of the quadriceps. ${ }^{26}$ The authors in these studies do not describe if participants also received usual rehabilitation interventions and their duration. Online supplemental appendix $\mathrm{C}$ provides further details of the interventions.

\section{Function and activity outcomes associated with health and social interventions for older adults and their caregivers after discharge from inpatient rehabilitation post-hip fracture}

Twenty-four intervention studies reported outcome measures using 29 different measurement tools, as some used more than one tool. Data are presented according to the primary outcomes reported by each of the study investigators. The conceptual framework for categorising geriatric rehabilitation outcomes developed by Demers et al guided the synthesis of the studies' outcomes. ${ }^{9}$ The outcomes in this review were divided into two major domains: (1) activity, which includes mobility, ADLs and IADL, and (2) functioning, which includes physical functioning and caregiver status. Table 3 lists the classification of the outcome measures outlined in the studies and the studies with significant results.

\section{Activity domain}

Mobility

Thirteen studies utilised mobility as the primary outcome. $^{30} 333436394043$ Mobility was measured using a variety of tests, such as the Timed-Up-and-Go Test in three studies, ${ }^{33} 3845$ Locomotion Subscale of the Functional Independence Measure,${ }^{34}$ New Mobility Score ${ }^{32} 33$ and the Short Physical Performance Battery (SPPB). ${ }^{23}$ Walking speed was measured using the 6 min Walk Test ${ }^{33} 4345$ and walking velocity. ${ }^{41}$ Mobility was measured using the gait speed test in three studies. ${ }^{23} 3039$ Walking ability and the use of walking device was measured in three studies. ${ }^{30} 3640$ Eight exercise-based studies found a significant change in mobility for participants. ${ }^{23} 33$ 34 $38-4145$ One non-exercisebased study reported no significant changes in the mobility score. ${ }^{26}$

\section{Activities of daily living}

Ten studies reported ADL as the primary outcome. ${ }^{19} 232629$ 36-38 404246 The following ADL measurement tools were used: Barthel Index (n=2), ${ }^{3740}$ Functional Independence Measure $(\mathrm{n}=2),{ }^{38} 44$ Katz Index $(\mathrm{n}=1),{ }^{42}$ Staircase of ADL, which measured ADLs using the Katz Index as well as IADLs $(\mathrm{n}=1),{ }^{36}$ Physical Function Performance $(n=1),{ }^{23}$ Chinese Barthel Index $(n=1)^{46}$ and a Physical Disability Questionnaire on perceived difficulties in performing 6 ADLs and 8 IADLs $(n=1){ }^{29}$ Four exercise-based interventions studies found a significant improvement in ADLs, ${ }^{36} 384046$ whereas four studies found no significant change. ${ }^{23} 293744$ Two studies with non-exercise-based interventions investigated ADLs as an outcome, but neither reported associated change in outcomes. ${ }^{2642}$

\section{Instrumental ADL}

IADLs was used as a primary outcome in four exercisebased studies, ${ }^{29} 363844$ using four different measures: Instrumental Activity Measure $(\mathrm{n}=1),{ }^{38}$ Assessment of Living Skills and Resources $(n=1),{ }^{44}$ Staircase of ADL, which measured ADLs using the Katz Index as well as IADLs $(\mathrm{n}=1),{ }^{36}$ and a Physical Disability Questionnaire on perceived difficulties in performing 6 ADLs and 8 IADLs $(n=1) .{ }^{29}$ Out of four exercise-based studies, only one study reported significant improvement in IADL, ${ }^{38}$ and three reported no change. ${ }^{293644}$

\section{Functioning domain}

Physical functioning

Physical functioning refers to the physical abilities required to perform the previously mentioned activity domain and includes measures of balance, endurance, comfort and others such as range of motion and muscle strength. ${ }^{9}$ table 3 illustrates the measurements of physical functioning.

Balance was used as the primary outcome in nine studies. 232527283338394546 It was measured using five different validated measures: rate of falls, ${ }^{25} 2728$ Tandem Test, ${ }^{23}{ }^{33}$ Falls Efficacy Scale, ${ }^{46}$ SPPB,${ }^{23}$ and Functional Reach Test. $^{45}$ The Timed-Up-and-Go Test, although usually a measure for mobility, was used as a measure of clinical gait and balance. ${ }^{39}$ Improved balance and fall reduction were reported in four exercise-based studies, ${ }^{2533} 3839$ and in one non-exercise-based study. ${ }^{28}$ In comparison, three exercise-based studies demonstrated no significant improvement in balance tests, ${ }^{2345} 46$ and one non-exercise-based study found no significant improvements in fall reduction. ${ }^{42}$

Endurance included physical role functioning, as measured by the Short Form-36, ${ }^{23} 2431$ and exercise behaviour, as measured by the Outcome Expectations for 
Self-efficacy for Walking/Exercise Scale. ${ }^{35}$ Two exercisebased studies reported significant outcomes on physical role functioning, ${ }^{24}$ and exercise behaviour. ${ }^{35}$ An exercisebased study showed no statistical change in physical functioning. ${ }^{23}$ A non-exercise-based study looking at the effects of social support showed no improvement in physical role functioning. ${ }^{31}$ Comfort was measured as healthrelated quality of life. Only one exercise-based study used health-related quality of life as one of the primary outcomes. ${ }^{41}$ This study found significant improvement in health-related quality of life.

Other physical abilities included muscle strength and range of motion. Muscle strength included measurements of knee extensor strength, lower extremity muscle strength, leg extensor power and hip flexor strength. ${ }^{23263338414345}$ Hip range of motion was measured in one study. ${ }^{41}$ Three exercise-based studies found a significant increase in muscle strength. ${ }^{33} 3845$ In contrast, three exercise-based studies, ${ }^{23} 4143$ and one non-exercisebased study, ${ }^{26}$ reported no significant change in muscle strength and range of motion. ${ }^{41}$

\section{Caregiver status}

Within 24 included studies in this review, only one exercise-based study examined caregiver status using the Caregiver Strain Index. ${ }^{37}$ The study examined the association between older adult's improvement in exercise training and the caregiver strain. The study reported no significant difference in caregiver strain.

\section{DISCUSSION}

This review identified 24 intervention studies focused on improving the activity and functioning of older adults post-hip fracture. Our study adds to the existing reviews examining exercise-based interventions ${ }^{1011}{ }^{13}$ by including non-exercise-based interventions and a view on the importance of both function and activity outcomes of older adults as well as caregiver outcomes needed to achieve a full recovery. Within these studies, there are some common findings to support possible practice and research implications going forward. Overall, we identified two distinct types of interventions: (1) those that included an exercise-based component $(n=19),{ }^{23-25} 293032-34$ 36-46 and (2) those that did not $(n=5) .{ }^{26-28} 3142$ We found that the majority of exercise-based interventions reported improvements in older adults' physical health and functional independence in ADLs, led to fewer falls and a lower use of a walking aid. This is consistent with other research findings, which have shown that exercise-based interventions improve recovery of function and independence in ADL. ${ }^{48} 49$

The majority of the 24 studies involved multiple components. These most often included exercise, ADL training and home assessment with modifications (table 2). Only seven studies had single-component interventions including, exercise-only, ${ }^{33-35} 37 \quad 39$ social support-only ${ }^{31}$ and nutrition-only ${ }^{42}$ interventions. However, considering recovery is a multifactorial concept, it is recommended that interventions focused on recovery need to be multipronged. This is in keeping with the Rehabilitative Care Best Practice Framework for Patients with Hip Fracture, ${ }^{50}$ which recommends multicomponent in-home rehabilitation care. Specific interventions may include fall prevention education and training to improve independence in self-care, transfers, ADLs, ambulation, balance and gait training, environmental modifications and progressive strengthening exercise programmes. ${ }^{50}$ This review suggests that additional components could be included in the framework: a focus on osteoporosis prevention, ${ }^{23} 2528$ nutrition ${ }^{23} 3646$ and social supports. ${ }^{2445}$

Given the heterogeneity in the clinical presentation of older adults with hip fractures, programmes tailored to a person's unique needs and circumstances may be required. Only seven of the studies in our review included a tailored programme, all of which demonstrated favourable results. ${ }^{23293037384145}$ Offering a cadre of interventions and making them available based on the individual needs of the older adult and their caregiver may be an important area for future consideration. The need to tailor interventions has been echoed in a systematic review of rehabilitation practices in post-hip fracture older adults ${ }^{49}$ and from the results of a qualitative study by Bruun-Olsen $e t a l .{ }^{51}$ Bruun-Olsen $e t$ al found that being in recovery after a hip fracture disrupted older adults' lives as loss of mobility and the impact of age was profoundly present and that interventions should be individually adjusted to each patient's needs and personal prerequisites to enhancing adherence to the intervention and thereby possibly improve outcomes. ${ }^{51}$

Demers $e t a l$ s framework of rehabilitation outcomes for older adults served as a useful guide to categorise outcomes in the reviewed studies as they all focused on functioning and activity domains. ${ }^{9}$ Most of the studies focused on activity outcomes vs function. However, according to the framework, a focus on both domains is required for recovery. For example, improvements in physical functioning (dexterity, balance, no pain, endurance) are necessary for the activity to flourish (mobility, ADL, IADL). Furthermore, our review also highlighted gaps within this body of evidence on outcomes that were not addressed according to the framework. Most notably, the current literature has significant gaps in terms of research focused on the caregiver. No studies had caregiver-focused interventions; however, one study reported changes in caregiver strain following an exercise-based intervention for older adults. ${ }^{37}$ This is of concern, as caregivers play a significant role in the general well-being and outcomes of older adults post-hip fracture recovery. ${ }^{52}$ More research is needed to determine the impact that caregiver burden and quality of life may have on post-hip fracture rehabilitation in older adults. This finding is consistent with a recent review, which suggests that social support is associated with an improvement in older adults' functional recovery. ${ }^{53}$ Support for caregivers is vital as they can assist older adults in maximising their rehabilitation potential. 
However, without support for the care partner, caregiver burden may arise, which has been associated with older adults' inability to reintegrate back to normal activities. ${ }^{52}$

Despite the known benefits of inpatient and homebased rehabilitation in Canada, it is disproportionately lower for older adults with dementia. About $10 \%$ of patients with dementia received home-based rehabilitation. ${ }^{1}$ In this study, a second clear literature gap pertained to individuals with cognitive impairment, as most $(75 \%)$ of the studies in this review excluded older adults with cognitive impairment. This is consistent with the systematic review by Chu et al, ${ }^{12}$ which found only three studies that evaluated outcomes of community-based post-hip fracture interventions for older adults with cognitive impairment. Providing rehabilitation for persons with cognitive impairment such as dementia is challenged by the lack of capacity to participate in rehabilitation and poor insight into following orders. ${ }^{54}$ This is a significant gap, both in knowledge and practice, given that persons with dementia are 2.7 times more likely to have a hip fracture than those without. ${ }^{55}$ Thus, more studies are needed to show what interventions are most effective in supporting this proportion of older adults, which would better aid decision-makers in resource allocation.

Our review also highlights the variation in outcomes and their measurements for this population. Twenty-six different main outcomes were identified across the included studies. This is similar to findings from a systematic review by Chudyk $e t a t^{49}$ in which they found that no consistent set of key outcomes or measures were used to assess recovery for persons post-hip fracture. The main outcome measurements used in this review were the Barthel Index for ADLs, gait speed and Timed-up-and-Go Test for mobility, Instrumental Activity Measure for IADL, and muscle strength for physical functioning. Recently published guidelines by the American Physical Therapy Association provide recommendations on measures that should be used in the postacute period to examine physical impairments, activity limitations and health-related quality of life. ${ }^{13}$ The use of consistent outcome measures in future research studies will allow for more reliable comparisons and analysis across studies, and yield more robust evidence on which to base practice recommendations and policy decisions.

\section{Strengths and limitations}

To our knowledge, this is the first review to summarise data following older adults receiving care in the community postinpatient rehabilitation after hip fracture surgery, with a focus on health and social interventions influencing function and activity outcomes. The current review used Demers et al $l$ s conceptual framework of geriatric rehabilitation to categorise the wide-range of intervention outcomes. Limitations of the review include searching only five databases for English published studies, which considered as a potential publication bias. Other interventions may exist in non-English published articles. Another limitation included the inability to examine the effect of the variation in interventions (dose, duration and provider) with outcomes achieved, notably because the main outcomes used across studies were inconsistent. Additionally, we were not able to evaluate whether single components of multicomponent interventions led to positive outcomes or if there is a synergistic, combined effect of multicomponent interventions. Therefore, making comparisons across the 24 included studies proved challenging, resulting in an inability to synthesise results statistically. Future research will need to include an in-depth analysis of effect sizes to understand intervention effectiveness better.

\section{Implications and future directions}

There is evidence to support exercise-based, multicomponent health interventions in supporting function and recovery after hip fracture, but a paucity of studies on social supports and interventions. There is a need for studies focusing on older adults with cognitive impairment, who make up a significant proportion of patients with hip fractures and who experience poorer outcomes over time. Future research examining intervention components tailored to older adults and their caregivers is needed, and research with rigorous methods and standardised outcome measurements is needed to synthesise findings related to intervention effectiveness. These studies are needed to guide recovery pathways for this vulnerable group going forward. An in-depth analysis of effect sizes to understand intervention effectiveness better is recommended for future research.

\section{Author affiliations}

${ }^{1}$ Trent/Fleming School of Nursing, Trent University, Peterborough, Ontario, Canada

${ }^{2}$ Toronto Rehabilitation Institute, University Health Network, Toronto, Ontario, Canada ${ }^{3}$ Lawrence S. Bloomberg Faculty of Nursing, University of Toronto, Toronto, Ontario,

Canada

${ }^{4}$ Nursing, University of Toronto Lawrence S Bloomberg Faculty of Nursing, Toronto, Ontario, Canada

${ }^{5}$ Rehabilitation Sciences Institute, Faculty of Medicine, University of Toronto, Toronto, Ontario, Canada

${ }^{6}$ Bruyère Research Institute, Ottawa, Ontario, Canada

${ }^{7}$ Ottawa Hospital Research Institute, Ottawa, Ontario, Canada

${ }^{8}$ Centre for Health and Safety in Agriculture, University of Saskatchewan College of Medicine, Saskatoon, Saskatchewan, Canada

${ }^{9}$ School of Epidemiology and Public Health, University of Ottawa, Ottawa, Ontario, Canada

${ }^{10}$ Department of Psychiatry, University of Toronto, Toronto, Ontario, Canada

Twitter Shirin Vellani @vellani_shirin

Acknowledgements KSM is supported by the Walter and Maria Schroeder Institute for Brain Innovation \& Recovery. We wish to acknowledge Doug Salzwedel for his assistance with the literature review and Howard Chow for his assistance with article screening and data extraction.

Contributors $\mathrm{AO}$ is responsible for the overall content as guarantor. Writingoriginal draft preparation: $\mathrm{AO}$ and $\mathrm{AC}$. Writing-review and editing: $\mathrm{SV}, \mathrm{AK}, \mathrm{Al}, \mathrm{JL}, \mathrm{MB}$, KSM, AO and AC. Data curation: SV and AK. Validation: VAW and EG. Supervision: KSM.

Funding This review was funded in part by a research grant from the Ontario Ministry of Health and Long-Term Care (MOHLTC) to the Ontario QUILT (Quality for Individuals who require Long-Term support) Network (Grant ID no. 255).

Competing interests None declared.

Patient consent for publication Not applicable. 
Provenance and peer review Not commissioned; externally peer reviewed.

Data availability statement Data are available on reasonable request. The extraction of the studies included in this review is available upon reasonable request.

Supplemental material This content has been supplied by the author(s). It has not been vetted by BMJ Publishing Group Limited (BMJ) and may not have been peer-reviewed. Any opinions or recommendations discussed are solely those of the author(s) and are not endorsed by BMJ. BMJ disclaims all liability and responsibility arising from any reliance placed on the content. Where the content includes any translated material, BMJ does not warrant the accuracy and reliability of the translations (including but not limited to local regulations, clinical guidelines, terminology, drug names and drug dosages), and is not responsible for any error and/or omissions arising from translation and adaptation or otherwise.

Open access This is an open access article distributed in accordance with the Creative Commons Attribution Non Commercial (CC BY-NC 4.0) license, which permits others to distribute, remix, adapt, build upon this work non-commercially, and license their derivative works on different terms, provided the original work is properly cited, appropriate credit is given, any changes made indicated, and the use is non-commercial. See: http://creativecommons.org/licenses/by-nc/4.0/.

\section{ORCID iDs}

Abeer Omar http://orcid.org/0000-0002-4713-2874

Shirin Vellani http://orcid.org/0000-0001-6032-0266

Alexandra Krassikova http://orcid.org/0000-0001-9828-1231

Vivian A Welch http://orcid.org/0000-0002-5238-7097

\section{REFERENCES}

1 Seitz DP, Gill SS, Austin PC, et al. Rehabilitation of older adults with dementia after hip fracture. J Am Geriatr Soc 2016;64:47-54.

2 Dyer SM, Crotty M, Fairhall N, et al. A critical review of the long-term disability outcomes following hip fracture. BMC Geriatr 2016;16:158.

3 Magaziner J, Hawkes W, Hebel JR, et al. Recovery from hip fracture in eight areas of function. J Gerontol A Biol Sci Med Sci 2000;55:M498-507.

4 McGilton KS, Vellani S, Zheng N, et al. Healthcare professionals' perspectives on rehabilitating persons with cognitive impairment. Dementia 2021;20:1772-90.

5 World Health OrganizationTeam. Rehabilitation 2030: a call for action. World Health organization, 2017. Available: https://www.who.int/ publications/m/item/rehabilitation-2030-a-call-for-action

6 Pitzul KB, Wodchis WP, Carter MW, et al. Post-Acute pathways among hip fracture patients: a system-level analysis. BMC Health Serv Res 2016;16:275

7 Intrator O, Berg K. Benefits of home health care after inpatient rehabilitation for hip fracture: health service use by Medicare beneficiaries, 1987-1992. Arch Phys Med Rehabil 1998;79:1195-9.

8 McGilton KS, Campitelli MA, Bethell J, et al. Impact of dementia on patterns of home care after inpatient rehabilitation discharge for older adults after hip fractures. Arch Phys Med Rehabil 2021;102:1972-81.

9 Demers L, Ska B, Desrosiers J, et al. Development of a conceptual framework for the assessment of geriatric rehabilitation outcomes. Arch Gerontol Geriatr 2004;38:221-37.

10 Wu D, Zhu X, Zhang S. Effect of home-based rehabilitation for hip fracture: a meta-analysis of randomized controlled trials. J Rehabil Med 2018;50:481-6.

11 Auais MA, Eilayyan O, Mayo NE. Extended exercise rehabilitation after hip fracture improves patients' physical function: a systematic review and meta-analysis. Phys Ther 2012;92:1437-51.

$12 \mathrm{Chu} \mathrm{CH}$, Paquin K, Puts M, et al. Community-Based hip fracture rehabilitation interventions for older adults with cognitive impairment: a systematic review. JMIR Rehabil Assist Technol 2016;3:e3.

13 McDonough CM, Harris-Hayes M, Kristensen MT, et al. Physical therapy management of older adults with hip fracture. J Orthop Sports Phys Ther 2021;51:CPG1-81.

14 Beaupre LA, Binder EF, Cameron ID, et al. Maximising functional recovery following hip fracture in frail seniors. Best Pract Res Clin Rheumatol 2013;27:771-88.

15 Arksey H, O'Malley L. Scoping studies: towards a methodological framework. Int J Soc Res Methodol 2005;8:19-32.

16 Levac D, Colquhoun H, O'Brien KK. Scoping studies: advancing the methodology. Implement Sci 2010;5:69.

17 Tricco AC, Lillie E, Zarin W, et al. PRISMA extension for scoping reviews (PRISMA-ScR): checklist and explanation. Ann Intern Med 2018;169:467-73.
18 Department of Health and Social Care. DoHaS. National Framework for NHS Continuing Healthcare \& NHS-funded Nursing Care: Incorporating the NHS Continuing Healthcare Practice Guidance, 2018. Available: https://assets.publishing.service.gov.uk/ government/uploads/system/uploads/attachment_data/file/746063/ 20181001_National_Framework_for_CHC_and_FNC_-_October_ 2018 Revised.pdf

19 SMea D. Rehabilitation Following Hip Fracture. In: Falaschi P, ed. Orthogeriatrics practical issues in geriatrics. Second ed. Cham: Springer, 2021.

20 Binder EF, Brown M, Sinacore DR, et al. Effects of extended outpatient rehabilitation after hip fracture: a randomized controlled trial. JAMA 2004;292:837-46.

21 Babineau J. Product review: Covidence (systematic review software) $J$ Can Health Libr Assoc 2014;35:68-71.

22 McHugh ML. Interrater reliability: the kappa statistic. Biochem Med 2012:22:276-82.

23 Adunsky A, Chandler J, Heyden N, et al. MK-0677 (ibutamoren mesylate) for the treatment of patients recovering from hip fracture: a multicenter, randomized, placebo-controlled phase llb study. Arch Gerontol Geriatr 2011;53:183-9.

24 Allegrante JP, Peterson MGE, Cornell CN, et al. Methodological challenges of multiple-component intervention: lessons learned from a randomized controlled trial of functional recovery after hip fracture. Hss J 2007;3:63-70.

25 Bischoff-Ferrari HA, Dawson-Hughes B, Platz A, et al. Effect of high-dosage cholecalciferol and extended physiotherapy on complications after hip fracture: a randomized controlled trial. Arch Intern Med 2010;170:813-20.

26 Braid V, Barber M, Mitchell SL, et al. Randomised controlled trial of electrical stimulation of the quadriceps after proximal femoral fracture. Aging Clin Exp Res 2008;20:62-6.

27 Di Monaco M, De Toma E, Gardin L, et al. A single postdischarge telephone call by an occupational therapist does not reduce the risk of falling in women after hip fracture: a randomized controlled trial. Eur J Phys Rehabil Med 2015;51:15-22.

28 Di Monaco M, Vallero F, De Toma E, et al. A single home visit by an occupational therapist reduces the risk of falling after hip fracture in elderly women: a quasi-randomized controlled trial. J Rehabil Med 2008;40:446-50.

29 Edgren J, Salpakoski A, Sihvonen SE, et al. Effects of a homebased physical rehabilitation program on physical disability after hip fracture: a randomized controlled trial. J Am Med Dir Assoc 2015;16:350.e1-7.

30 Karlsson Åsa, Berggren M, Gustafson Y, et al. Effects of Geriatric Interdisciplinary Home Rehabilitation on Walking Ability and Length of Hospital Stay After Hip Fracture: A Randomized Controlled Trial. $J$ Am Med Dir Assoc 2016;17:464.e9-464.e15.

31 Kirk-Sanchez NJ. The importance of psychosocial factors in determining disability and perceived health in a group of older Hispanic-Americans recovering from hip fracture. $J$ Geriatr Phys Ther 2003;26:3-8.

32 Magaziner J, Mangione KK, Orwig D, et al. Effect of a multicomponent home-based physical therapy intervention on ambulation after hip fracture in older adults: the cap randomized clinical trial. JAMA 2019;322:946-56.

33 Overgaard J, Kristensen MT. Feasibility of progressive strength training shortly after hip fracture surgery. World $J$ Orthop 2013;4:248-58.

34 Penrod JD, Boockvar KS, Litke A, et al. Physical therapy and mobility 2 and 6 months after hip fracture. J Am Geriatr Soc 2004:52:1114-20.

35 Resnick B, Orwig D, Yu-Yahiro J, et al. Testing the effectiveness of the exercise plus program in older women post-hip fracture. Ann Behav Med 2007:34:67-76.

36 Stenvall M, Olofsson B, Nyberg L, et al. Improved performance in activities of daily living and mobility after a multidisciplinary postoperative rehabilitation in older people with femoral neck fracture: a randomized controlled trial with 1-year follow-up. $J$ Rehabil Med 2007;39:232-8.

37 Williams NH, Roberts JL, Din NU, et al. Fracture in the elderly multidisciplinary rehabilitation (femur): a phase II randomised feasibility study of a multidisciplinary rehabilitation package following hip fracture. BMJ Open 2016;6:e012422.

38 Zidén L, Frändin K, Kreuter M. Home rehabilitation after hip fracture. A randomized controlled study on balance confidence, physical function and everyday activities. Clin Rehabil 2008;22:1019-33.

39 Kalron A, Tawil H, Peleg-Shani S, et al. Effect of telerehabilitation on mobility in people after hip surgery: a pilot feasibility study. Int $J$ Rehabil Res 2018;41:244-50. 
40 Pourabbas B, Emami MJ, Vosoughi AR, et al. Does mobility of the elderly with hip fractures improve at one year following surgery? A 5-year prospective survey. Ortop Traumatol Rehabil 2016;18:311-6.

41 Tsauo J-Y, Leu W-S, Chen Y-T, et al. Effects on function and quality of life of postoperative home-based physical therapy for patients with hip fracture. Arch Phys Med Rehabil 2005;86:1953-7.

42 Bruce D, Laurance I, McGuiness M, et al. Nutritional supplements after hip fracture: poor compliance limits effectiveness. Clin Nutr 2003;22:497-500.

43 Moseley AM, Sherrington C, Lord SR, et al. Mobility training after hip fracture: a randomised controlled trial. Age Ageing 2009;38:74-80.

44 Singh NA, Quine S, Clemson LM, et al. Effects of high-intensity progressive resistance training and targeted multidisciplinary treatment of frailty on mortality and nursing home admissions after hip fracture: a randomized controlled trial. J Am Med Dir Assoc 2012;13:24-30.

45 Peterson MGE, Ganz SB, Allegrante JP, et al. High-Intensity exercise training following hip fracture. Top Geriatr Rehabil 2004;20:273-84.

46 Shyu Y-IL, Liang J, Tseng M-Y, et al. Comprehensive care improves health outcomes among elderly Taiwanese patients with hip fracture. J Gerontol A Biol Sci Med Sci 2013;68:188-97.

47 Langford DP, Fleig L, Brown KC, et al. Back to the future - feasibility of recruitment and retention to patient education and telephone follow-up after hip fracture: a pilot randomized controlled trial. Patient Prefer Adherence 2015;9:1343-51.
48 Beaupre LA, Magaziner JS, Jones CA, et al. Rehabilitation after hip fracture for nursing home residents: a controlled feasibility trial. $J$ Gerontol A Biol Sci Med Sci 2019;74:1518-25.

49 Chudyk AM, Jutai JW, Petrella RJ, et al. Systematic review of hip fracture rehabilitation practices in the elderly. Arch Phys Med Rehabil 2009;90:246-62.

$50 \mathrm{RC}$ A. Rehabilitative care best practices for patients with hip fracture, 2018.

51 Bruun-Olsen V, Bergland A, Heiberg KE. "I struggle to count my blessings": recovery after hip fracture from the patients' perspective. BMC Geriatr 2018;18:18.

52 McGilton KS, Omar A, Stewart SS, et al. Factors that influence the reintegration to normal living for older adults 2 years post hip fracture. J App/ Gerontol 2020;39:1323-31.

53 Auais M, Al-Zoubi F, Matheson A, et al. Understanding the role of social factors in recovery after hip fractures: a structured scoping review. Health Soc Care Community 2019;27:1375-87.

54 Cations M, May N, Crotty M, et al. Health professional perspectives on rehabilitation for people with dementia. Gerontologist 2020;60:503-12.

55 Hall AJ, Lang IA, Endacott R, et al. Physiotherapy interventions for people with dementia and a hip fracture-a scoping review of the literature. Physiotherapy 2017;103:361-8. 